\title{
Nearly Optimal Three Dimensional Layout of Hypercube Networks
}

\author{
Tiziana Calamoneri and Annalisa Massini \\ Department of Computer Science, University of Rome "La Sapienza" \\ - Italy, via Salaria 113, 00198 Roma, Italy. \\ massini@dsi.uniroma1.it
}

\begin{abstract}
In this paper we consider the three-dimensional layout of hypercube networks. Namely, we study the problem of laying hypercube networks out on the three-dimensional grid with the properties that all nodes are represented as rectangular slices and lie on two opposite sides of the bounding box of the layout volume. We present both a lower bound and a layout method providing an upper bound on the layout volume of the hypercube network.
\end{abstract}

Keywords: Hypercube Network, Three Dimensional Layout, VLSI layout volume.

\section{Introduction}

The layout of interconnection networks has important cost and performance implications for multiprocessors and parallel and distributed systems based on components. Thus, there is currently renewed interest in finding efficient VLSI layouts for various interconnection networks (see for instance [2,4,9,13,14,15,16]).

Recent hardware advances have allowed three-dimensional circuits to have a cost low enough to make them commonly available. For this reason threedimensional layouts of interconnection topologies on rectilinear grids are becoming of wide interest. The importance of efficiently representing interconnection networks in three dimensions has already been stated in the 80's by Rosenberg [11, and the most relevant aims are to shorten wires and to save in material (i.e. to minimize volume). The three-dimensional layout problem is related both with the study of the VLSI layout problem for integrated circuits and with the study of algorithms for drawing graphs. Indeed, the tie between VLSI layout studies and theoretical graph drawing is very strong since laying out a network on a grid is equivalent to orthogonally drawing the underlying graph, except for the fact that wire crossings are allowed. Nevertheless, almost all the results known in the literature about three-dimensional grid drawing of graphs are valid for very general graphs (see, the proceedings of the past editions of the Graph Drawing Symposia and, for instance, [11512]) and therefore they do not work efficiently for structured and regular graphs such as the most commonly used interconnection topologies, either the produced volume is too large (in order of 
magnitude or in the multiplicative constant), or the length of each wire is not kept of the same order of magnitude and sufficiently small, or the shape of nodes is not feasible to represent a processor, a switch, etc.. Hence, usually, lower and upper bounds on the layout volume of specific interconnection topologies must be established and the layout technique is as more good as these bounds are closer; of course, the layout method should try to highlight all the interesting properties of the network and to make as fast as possible the flow through it by shortening the maximum wire length.

The hypercube network has been widely considered as a network for parallel computing for its good parameters, e.g. regularity, logarithmic diameter, fault tolerance. A further parameter distinguishing 'good' networks is whether they can be laid out compactly in the orthogonal grid. This issue has already been studied in two dimensions [6]. In this paper we study the three-dimensional layout of the hypercube networks with the property that nodes lie on two planes, corresponding to opposite sides of the bounding box, partitioned into two equally sized sets, and they are represented as rectangular slices. The model of keeping all nodes on the surface of the bounding box is motivated by two main reasons: (i) it decreases the problems due to power consumption and concomitant heat generation and (ii) it makes easier the connection of the topologies to other part of the architecture, as the wires are necessarily connected to nodes. We present upper and lower bounds for the layout volume of the hypercube networks that are very close, although not coinciding (in fact they differ by a factor of $\log ^{1 / 2} N$ ). The maximum wire-length of the presented layout is $O(\log N \sqrt{N})$.

The rest of this paper is organized as follows: in Section 2 we give some preliminary definitions and recall some useful previous results. Section 3 is the core of the work and we present both a lower bound and a layout method providing an upper bound on the layout volume of the hypercube network. Finally, in Section 4 we address some conclusions and list some interesting open problems.

\section{Preliminaries}

In this section we recall some useful definitions and state some preliminary results.

A hypercube $Q_{n}$ of dimension $n$ is an interconnection network with $N=2^{n}$ nodes, each labeled with an $n$-bit binary string. Two nodes are connected by an edge if their labels differ in exactly one bit.

It is easy to see that a $Q_{n}$ can be constructed by considering two copies of $Q_{n-1}$, adding an edge for each pair of equally labeled nodes and completing the labels of nodes by adding one bit ( 0 in the first copy and 1 in the second copy of $\left.Q_{n-1}\right)$. In the following we will call homonym nodes in $Q_{n}$ two nodes connected by this inductive step, i.e. two nodes whose labels differ only in the leftmost bit.

By iterating this recursive construction, we deduce that $Q_{n}$ can be constructed starting from $2^{n-4}$ copies of $Q_{4}$, adding some edges and readjusting the labels. In the following we will extensively use this observation and we will clarify the reason why $Q_{4}$ is chosen. 
Another interesting property of $Q_{n}$ we will exploit in the following is its bipartiteness; a feasible partition of the nodes can be obtained by dividing nodes having an even number of 0 s in their label from nodes having an odd number of 0s in their label. The bipartiteness property is particularly evident when $Q_{n}$ is visualized as a lattice (see Fig. 1).

Finally, observe that the hypercube $Q_{n}$ is a regular graph of degree $n$, so it is not possible to represent nodes as dots for each $n>6$.

A three-dimensional grid layout $\Gamma(\mathcal{G})$ of an interconnection network $\mathcal{G}$ is a mapping of $\mathcal{G}$ to the three-dimensional grid such that nodes are mapped to gridnodes and edges are mapped to independent grid-paths satisfying the following conditions:

- distinct grid-paths are edge-disjoint (then at most three paths can cross at a grid-node);

- grid-paths that share an intermediate grid-node must cross at that node (that is 'knock-knee' paths [8] are not allowed);

- a grid-path may touch no mapped node, except at its end-points.

If $\Gamma(\mathcal{G})$ can be enclosed in a $H \times W \times L$ three-dimensional box, the volume of $\Gamma(\mathcal{G})$ is the product $H \times W \times L$.

We define the $a \times b$-3D double channel routing as a slight modification of the concept of $k$-3D channel routing introduced in [3. To this aim, we need to recall the following definition:

Definition 1. A $k$-channel routing involves a bidimensional grid and two sets $S$ and $S^{\prime}$ each consisting of $k$ nodes to be connected by a 1-1 function. $S$ and $S^{\prime}$ are arranged onto opposite sides of the grid.

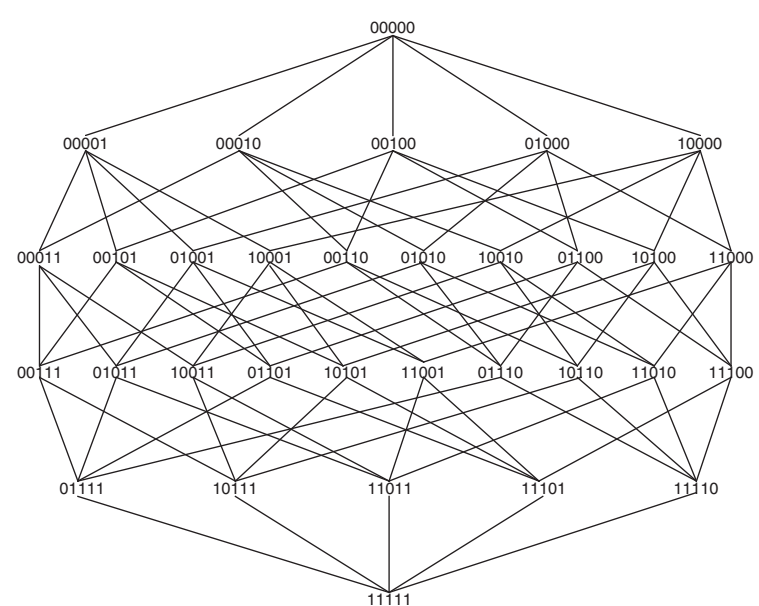

Fig. 1. $Q_{5}$ represented as a lattice. 
The grid involved in any $k$-channel routing is not greater than $(k+1) \times\left(\frac{3}{2} k+2\right)$ and $S$ and $S^{\prime}$ lie on the shortest sides, as shown in [10].

Definition 2. An $a \times b$-3D double channel routing involves a three-dimensional grid (the channel) and two sets $S$ and $S^{\prime}$, each containing $a \cdot b$ nodes, arranged on the grid nodes of opposite faces of dimension $a \times b$, to be connected by $a$ 1-1 function $f$. Function $f$ associates to a node $(x, y)$ of $S$ a node $\left(x^{\prime}, y^{\prime}\right)$ of $S^{\prime}$ such that $x^{\prime}=g(x)$ and $y^{\prime}=h(y)$, where functions $g$ and $h$ are two-dimensional $a-$ and $b$-channel routings, respectively.

In the following, for the sake of brevity, when no confusion arises, we refer to the $a \times b$-3D double channel routing as $3 D$ channel routing.

The following theorem states the necessary volume for any $3 \mathrm{D}$ channel routing. In this preliminary version of the paper we omit its proof.

Theorem 1. A three-dimensional grid of size $(a+1) \times(b+1) \times \max \left\{\frac{3}{2} a+\right.$ $\left.2, \frac{3}{2} b+2\right\}$ is enough to realize a $3 D$ channel routing.

\section{Three Dimensional Layout of Hypercube Network}

In this section we discuss the problem of laying the hypercube network out; in particular, we determine lower and upper bounds for the volume.

\subsection{Lower Bound}

In order to compute a lower bound on the layout volume of the hypercube network, we can use the general formula provided in [3]:

$$
\text { lower bound on the layout volume of } \mathcal{H} \geq(\sqrt{B W(\mathcal{H})}-1)^{3}
$$

where $\mathcal{H}$ is a general network and $B W(\mathcal{H})$ is its bisection width.

It is well known that the bisection width of $Q_{n}$ is $\frac{N}{2}[7]$, so a lower bound for the layout volume for $Q_{n}$ is $\Omega\left(N^{\frac{3}{2}}\right)$. One can object that this lower bound does not take into account the fact that, when $n>6$, nodes cannot be represented as dots (i.e. with null area), but it is necessary to use a space (either area or volume) proportional to the degree for each of them. Furthermore, the previous formula assumes that the nodes can be distributed anywhere inside the volume of the layout. On the contrary, our aim is to find a three-dimensional layout of $Q_{n}$ with some special properties; namely, all nodes are represented as rectangular slices with area proportional to their degree, they must lie on two planes, corresponding to opposite sides of the bounding box, and they are partitioned into two equally sized sets. Under these assumptions, we can better specify the value of the lower bound on the volume as $\Omega\left(N^{3 / 2} \log ^{1 / 2} N\right)$.

We compute this lower bound by exploiting a proof technique presented in [11] for one-active-level layouts. Let $W, L$ and $H$ be the dimensions of the bounding box of any three-dimensional layout of $Q_{n}$ with nodes partitioned into two equally sized sets and laid out on two opposite sides. Without loss of 
generality, assume the nodes lie on the sides of dimension $W \times L$ of the bounding box. Since each side contains $\frac{N}{2}$ nodes, each of degree $\log N$, it is straightforward to see that $W \times L=\Omega\left(\frac{N}{2} \log N\right)$. Two cases can occur, according to the fact that $H \geq L, W$ or not.

If $H \geq L, W$, then $H \geq\left(\frac{N}{2} \log N\right)^{1 / 2}$. It follows that the volume must be $\Omega\left(\left(\frac{N}{2}\right)^{3 / 2} \log ^{3 / 2} N\right)$, which is even greater than we claim.

If $H$ is not the maximum dimension of the bounding box, we can consider a plane $\pi$ orthogonal to the sides of the bounding box containing nodes and parallel to the pair of parallel sides of the bounding box of dimension $L \times H$. We place $\pi$ in such a way that $\pi$ cuts the layout into two equal portions. The intersection of the bounding box with plane $\pi$ has area $L \times H$ and $\pi$ partitions each set of nodes into two equally sized sets leaving $\frac{N}{2}$ nodes to its left $\left(\frac{N}{4}\right.$ on the upper face and $\frac{N}{4}$ on the lower face of the bounding box) and $\frac{N}{2}$ nodes to its right $\left(\frac{N}{4}\right.$ on the upper face and $\frac{N}{4}$ on the lower face of the bounding box). Hence, $\pi$ cuts a number of edges at least equal to the bisection width of $Q_{n}$, i.e. $\frac{N}{2}$. It follows that the area of $\pi$ is at least $\frac{N}{2}$, i.e. $H \times L \geq \frac{N}{2}$. Since $L \geq N^{1 / 2} \log ^{1 / 2} N$ we have that the volume is $\Omega\left(N^{3 / 2} \log ^{1 / 2} N\right)$.

The previous considerations lead to the following:

Theorem 2. A lower bound for a $3 D$ grid layout of $Q_{n}$ is $\Omega\left(N^{3 / 2} \log ^{1 / 2} N\right)$, if the layout has the following properties:

- all nodes are represented as rectangular slices with area proportional to their degree,

- all nodes lie on two planes, corresponding to opposite sides of the bounding box,

- nodes are partitioned into two equally sized sets.

\subsection{Layout of $Q_{n}$}

In this subsection we provide a method to lay the hypercube network out for each $n \geq 4$ (indeed, for $n=3$ the layout is trivial).

In our layout we choose to represent nodes as rectangular slices of area $1 \times$ $\left\lceil\frac{n}{2}-1\right\rceil$ (see Fig. 2). Each dot at integer coordinates on the slice is a pin where one wire is connected. Observe that when $n$ is odd, the last column of each slice has only one active pin, whereas when $n$ is even, all pins of the slice are active (see Fig. 21).

As we highlighted in the previous section, the basic building box of our result is the layout of $Q_{4}$, so we show it, before generalizing the construction to $Q_{n}$.

Since the degree of nodes in $Q_{4}$ is 4 , we represent each node as a unit area slice and we arrange these slices in two rectangles of dimension $3 \times 7$, as shown in Fig. 3] Our layout puts nodes onto opposite sides of the bounding box, and all edges are routed inside. Hence, we exploit the property that $Q_{4}$ is bipartite and put on one side (let it be the upper side) all nodes having an even number of 0 s in their label, and on the opposite side (let it be the lower side) the remaining nodes (see Fig. 3). 


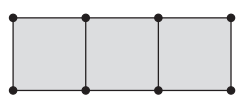

a.

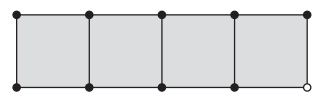

b.

Fig. 2. Slices representing nodes of degree $n$; a. $n$ even; b. $n$ odd; the white dot is not active.

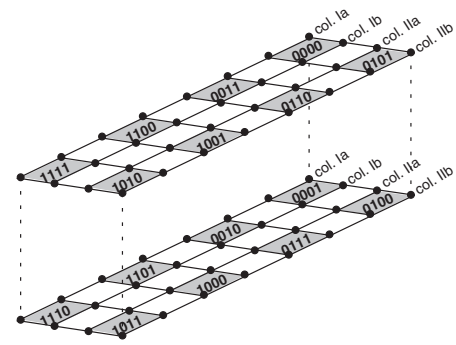

Fig. 3. Placement of $Q_{4}$ 's nodes on a three-dimensional grid.

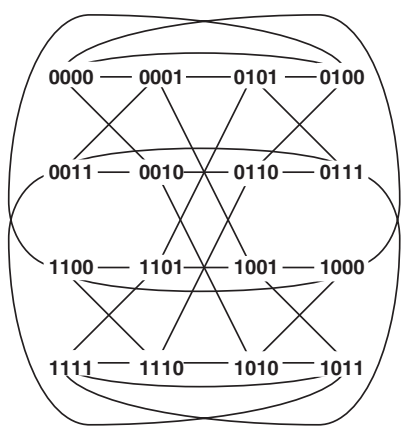

Fig. 4. $Q_{4}$ represented as a butterfly-like network.

Furthermore, we want to use a 3D channel routing to arrange all edges on the grid. To this aim, we need to assign to each node a position in such a way that the rules of 3D channel routing (cf. Def. 2) are maintained; namely, we have to map columns of the upper side to columns of the lower side. In other words, all nodes of each column $i$ on the upper side must be mapped to nodes of a column $j$ on the lower side. The way how to arrange nodes on the rectangle is highlighted from the representation of $Q_{4}$ shown in Fig. 4 . Indeed, we can map the leftmost node column of Fig. 4 to the first column of the upper side (see Fig. 3), the second node column in Fig. 4 in the first column of the lower side, and so on. Of course, we use pins in the same subcolumn (either $a$ or $b$ ) to layout edges outgoing from each node and going towards right (left) in Fig. 4 
Such an assignment respects the rules of a 3D channel routing, and a possible final three-dimensional layout is sketched in Fig. 5 where the 4-channel routings inside the bent planes are not drawn for the sake of clarity. The volume of the built layout for $Q_{4}$ is $8 \times 4 \times 5$.

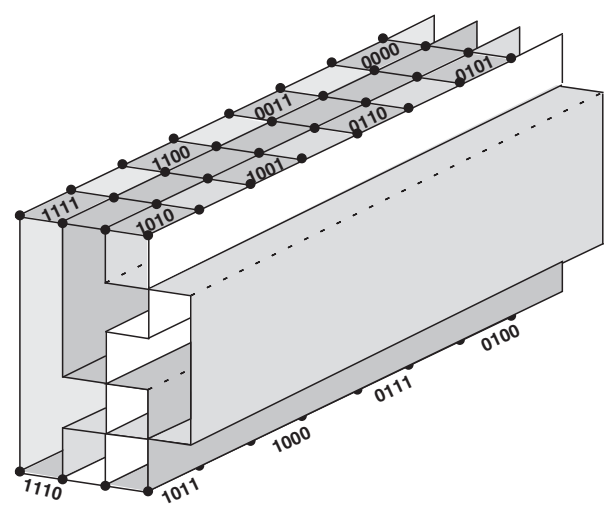

Fig. 5. 3D layout of $Q_{4}$, where the 4-channel routings inside the bent planes are not drawn.

In order to generalize our layout to each $n \geq 4$, we exploit the layout of $Q_{4}$ and the recursive definition of $Q_{n}$. Namely, given a hypercube $Q_{n}$, we will arrange it on the three-dimensional grid as $2^{n-4}$ copies of hypercubes of dimension 4 plus a set of edges. We claim that this set of edges constitutes a 3D channel routing.

We give the construction and the proof of our claim by induction on $n$.

Base. The claim is trivially true when $n=4$.

Inductive Hypothesis. $Q_{r}, 4 \leq r<n$, can be arranged on the grid as $2^{r-4}$ copies of $Q_{4}$ s plus a set of edges constituting a 3D channel routing.

Inductive Step. $Q_{n}$ can be obtained by considering two copies of $Q_{n-1}$ and connecting with an edge each pair of homonym nodes. In view of the inductive hypothesis, the claim is true for $Q_{n-1}$. So, we can represent $Q_{n}$ on the threedimensional grid by putting two copies of 3D layouts of $Q_{n-1}$ s one beside the other. Since the copies of $Q_{n-1} \mathrm{~s}$ consist of $2^{n-5}$ copies of $Q_{4} \mathrm{~s}$ each by inductive hypothesis, $Q_{n}$ will consist of $2^{n-4}$ copies of $Q_{4}$ s. It remains to prove that the edges connecting homonym pairs plus the existing $3 \mathrm{D}$ channel routings inside the two copies of $Q_{n-1}$ constitute a new 3D channel routing.

Let us distinguish two different cases according to the parity of $n$ :

\section{$-n$ odd}

Arrange the 3D layouts of $Q_{n-1} \mathrm{~S}$ one beside the other but swapping upper and lower sides in one of them, in such a way that homonym nodes lie on opposite sides of the bounding box (see Fig. 6. a). 

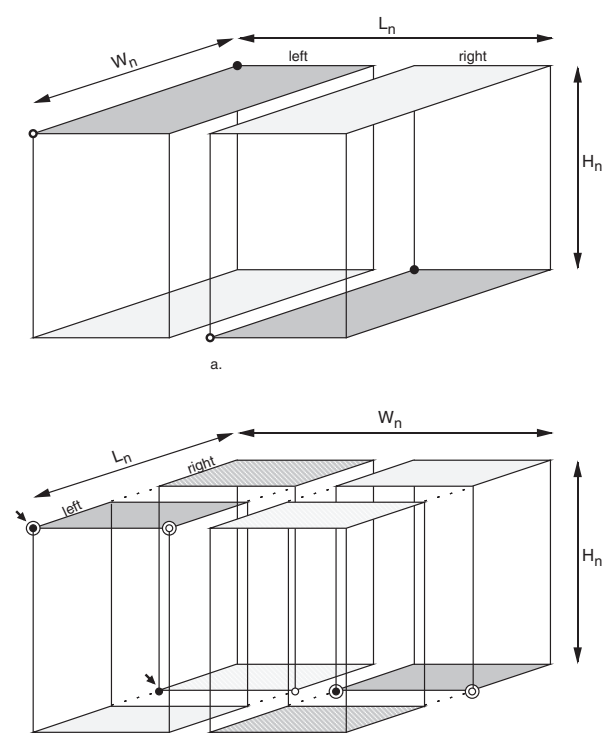

b.

Fig. 6. Construction of $Q_{n}$ starting from two copies of $Q_{n-1}$ s; a. $n$ odd; b. $n$ even. Double circled nodes represent homonym nodes in the two copies of $Q_{n-1}$ s. Simply circled nodes are homonym nodes of those in the upper part of the layout at the previous step, i.e. in the two copies of $Q_{n-2}$ s inside the same copy of $Q_{n-1}$.

Observe that the set of edges connecting the copies of $Q_{4} \mathrm{~s}$ is a $3 \mathrm{D}$ channel routing. Indeed, it is constituted by a set of edges (inside the two copies of $\left.Q_{n-1} \mathrm{~s}\right)$ and by a set of edges connecting homonym nodes of the two copies of $Q_{n-1} \mathrm{~s}$. The first set forms two disjoint 3D channel routings by inductive hypothesis; the second set connects homonym nodes located on the upper side of the left (right) $Q_{n-1}$ with nodes located on the lower side of the right (left) $Q_{n-1}$ (for an example, see nodes in Fig. 6]a).

Each node has the last column constituted by only one active pin and it corresponds to the edge that must be still laid out. Hence, the second set is completely disjoint from the other one and it has even stronger properties than a $3 \mathrm{D}$ channel routing, namely it ordinately connects nodes of the same row with nodes on the same row and nodes on the same column with nodes on the same column.

From the previous observations it follows that these three $3 \mathrm{D}$ channel routings form all together a unique 3D channel routing.

- $n$ even

In this case each node has all columns constituted by two pins and the lower pin in the last column corresponds to the edges to be still laid out (see Fig. 2). The upper pin in the last column corresponds to edges laid out in the previous step (when connecting two copies of $Q_{n-2} \mathrm{~s}$ in a $Q_{n-1}$ ). Hence, 
when we route the edges connecting homonym nodes using the lower pins, we must take into account the existing 3D channel routing using the upper pins. Again, arrange the 3D layouts of $Q_{n-1}$ s one beside the other, swapping upper and lower sides in one of them and swapping also the order of its two copies of $Q_{n-2}$ s inside, as shown in Fig. 6 b.

This arrangement guarantees that the rules of the $3 \mathrm{D}$ channel routing are respected by the set of all edges connecting the copies of $Q_{4}$ s. Actually, this set is constituted by a set of edges inside the two copies of $Q_{n-1}$ s and a set of edges connecting homonym nodes of the two copies of $Q_{n-1}$ s. The first set forms two disjoint 3D channel routings by inductive hypothesis; inside this set, we want to highlight the subset of edges outgoing from the upper pin of the last column of each node. In view of the $3 \mathrm{D}$ channel routing of the previous step $n-1$ (odd), they connect each node in general position $(i, j)$ of the upper face, left (right) side of each copy of $Q_{n-1}$ with its homonym in position $(i, j)$ of the lower face, right (left) side of the same copy (for an example, see nodes pointed by the arrows in Fig. 6b). Now consider the second set: it connects each node in general position $(i, j)$ of the upper face, left (right) side of the first copy, with its homonym in position $(i, j)$ of the lower face, right (left) side of the second copy by means of the lower pin of the last column (for an example, see double circled nodes in Fig. 6.b). So all connections coming out from the last column of the slice go to the same column and we have a $3 \mathrm{D}$ channel routing.

\subsection{Upper Bound}

Starting from the construction of the layout of $Q_{n}$ presented in the previous subsection, we deduce an upper bound on the volume of the layout of $Q_{n}$.

Theorem 3. There exists a three-dimensional layout of $Q_{n}$ having volume $\Theta\left(N^{3 / 2} \log N\right)$.

Proof. Let $W_{n}, L_{n}$ and $H_{n}$ be the three dimensions of the bounding box of the layout of $Q_{n}$ we presented in the previous subsection. We define some recurrence equations in order to compute these values for each $n \geq 4$.

If $n=4$ we have already obtained $W_{4}=8, L_{4}=4$ and $H_{4}=5$ (see Fig. 5), and we use these values as base of the recurrence.

As our layout method is conceived, the equations are different according to $n$ odd or even.

If $n$ is odd, we have:

$W_{n}$ : The width of the layout of $Q_{n}$ is the same as the width of $Q_{n-1}$ (see Fig. 6]a), hence $W_{n}=W_{n-1}$.

$L_{n}: L_{n}=2 L_{n-1}+\sqrt{N / 2}$ as we use two copies of $Q_{n-1}$ s and we need to enlarge the slices constituting nodes by one column. The contribution of $\sqrt{N / 2}$ follows from the way of positioning the slices. 
$H_{n}$ : The height is increased by the $3 \mathrm{D}$ channel routing introduced to connect the two copies. As this $3 \mathrm{D}$ channel routing has $a=b=\sqrt{N / 2}$, its contribution to the height is $\frac{3}{2} \sqrt{N / 2}+2$, hence $H_{n}=H_{n-1}+\frac{3}{2} \sqrt{N / 2}+2$.

If $n$ is even, we have:

$W_{n}: W_{n}=2 W_{n-1}$ as we use two copies of $Q_{n-1} \mathrm{~s}$.

$L_{n}$ : The length of the layout of $Q_{n}$ is the same as the length of $Q_{n-1}$ (see Fig. 6] b), hence $L_{n}=L_{n-1}$. Indeed, the newly inserted edges do not introduce any new contribution to this dimension.

$H_{n}$ : Also in this case the height is increased by the $3 \mathrm{D}$ channel routing introduced to connect the two copies and $a=2 \sqrt{N / 2}$ and $b=\sqrt{N / 2}$. Nevertheless, the contribution to the height is due only to the connections outgoing from the lower pins of the last columns in the slices. Indeed, the contribution of the other connections has been already computed in $H_{n-1}$. Hence, we have to add only the difference between the contributions of the two 3D channel routings, i.e. $H_{n}=H_{n-1}+\frac{3}{2} \sqrt{N / 2}$.

By solving the previous recurrence equations, we get:

$$
\begin{gathered}
W_{n}=\Theta(\sqrt{N}) \\
L_{n}=\Theta(\sqrt{N} \log N) \\
H_{n}=\Theta(\sqrt{N})
\end{gathered}
$$

both when $n$ is even and when $n$ is odd.

It follows that the volume is $\Theta\left(N^{\frac{3}{2}} \log N\right)$.

Observe that this upper bound differs from the lower bound only by a $\log ^{1 / 2} N$ factor.

Lemma 1. The $3 D$ layout of $Q_{n}$ presented in the previous section has maximum wire length $O(\sqrt{N} \log N)$.

Proof. It is easy to see that the maximum wire length can be computed considering the worst case in the $3 \mathrm{D}$ channel routing inside the layout.

As highlighted in Fig. 7 a, each plane constituting the 3D channel routing is at most $H_{n}+2 L_{n}-1$ long. Inside this plane (see Fig. $7 \mathrm{~b}$ ), the longest wire is $H_{n}+2 L_{n}-1+2 W_{n}-1$.

From this formula and from the values of $H_{n}, W_{n}$ and $L_{n}$ found in the previous proof, the claim follows.

Observe that in the two-dimensional layout of $Q_{n}$, the maximum wire length is $\Theta(N)[6]$, so this 3D layout saves both in material and in wire-length.

We conclude this subsection by summarizing all the results in the following theorem:

Theorem 4. The method described in the previous subsections provides a $3 D$ grid layout of $Q_{n}$ in $\Omega\left(N^{3 / 2} \log N\right)$ volume, with $O(\sqrt{N} \log N)$ maximum wire length, if the layout has the following properties: 


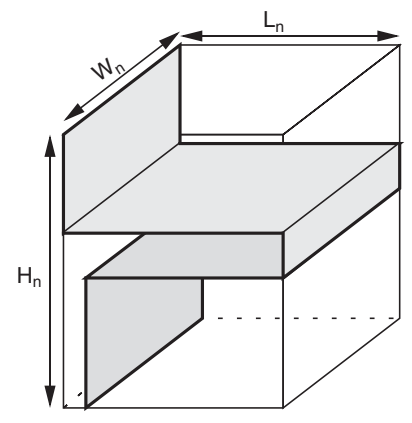

a.

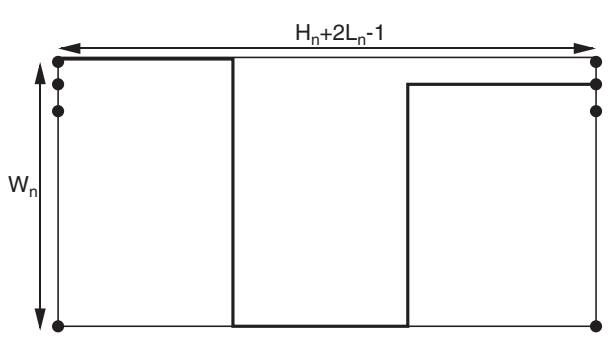

b

Fig. 7. a. A plane of the $3 \mathrm{D}$ channel routing inside the layout of $Q_{n}$ and b. a wire inside the same plane.

- all nodes are represented as rectangular slices with area proportional to their degree,

- all nodes lie on two planes, corresponding to opposite sides of the bounding box,

- nodes are partitioned into two equally sized sets.

\section{Conclusions and Open Problems}

In this paper we have considered the three-dimensional layout of hypercube networks. Namely, we have studied the problem of laying hypercube networks out on the three-dimensional grid with the properties that all nodes are represented as rectangular slices and lie on two opposite sides of the bounding box of the layout volume. We have presented upper and lower bounds for the layout volume of the hypercube networks that are very close, although not coinciding (in fact they differ by a factor of $\left.\log ^{1 / 2} N\right)$. The maximum wire-length of the presented layout is $O(\log N \sqrt{N})$.

Two open problems arise from this work. The first problem derives from the not coinciding upper and lower bounds, and consists in understanding which bound is tight. Secondly, it would be interesting to study the more general 3D layout problem, i.e. the problem where nodes are not constrained to be on two opposite sides of the bounding box but wherever inside the volume. In this case, it is possible to consider also other shapes for nodes (i.e. boxes). Concerning this problem, note that it is quite easy to find a $O\left(N^{3 / 2}\right)$ volume 3 D layout of $C C C_{n}$ network (i.e. the network obtained from $Q_{n}$ by substituting each $Q_{n}$ 's node with a cycle of length $n$ ) from the technique used in [3]. We have wondered if it is possible to deduce a $3 \mathrm{D}$ layout of $Q_{n}$ from the layout of $C C C_{n}$ with the same volume exploiting the definition of $C C C_{n}$. This does not seem possible, because the nodes constituting the cycles are not clustered in the layout. 


\section{References}

1. T. Biedl, T. Thiele, D.R. Wood: Three-Dimensional Orthogonal Graph Drawing with Optimal Volume. Proc. Graph Drawing (GD'00), pp. 284-295, 2001.

2. T. Calamoneri, A. Massini: An Optimal Layout of Multigrid Networks. Information Processing Letters, 72, pp. 137-141, 1999.

3. T. Calamoneri, A. Massini: Optimal three-dimensional layout of interconnection networks. Theoretical Computer Science, 255, pp. 263-279, 2001.

4. A. DeHon: Compact, Multilayer Layout for Butterfly Fat-Tree. ACM Symp. on Parallel Algorithms and Architectures (SPPA), 2000.

5. P. Eades, A. Symvonis, S. Whitesides: Two Algorithms for Three Dimensional Orthogonal Graph Drawing. Proc.Graph Drawing '96 (GD '96), Lectures Notes in Computer Science 1190, Springer-Verlag ,1996, pp 139-154.

6. R.I. Greenberg and L. Guan: On the area of hypercube layouts. Information Processing Letters, 84, pp. 41-46, 2002.

7. F.T. Leighton: Introduction to Parallel Algorithms and Architectures: Arrays, Trees and Hypercubes, Morgan Kaufmann Publ., San Mateo, CA, 1992.

8. K. Melhorn, F.P. Preparata and M. Sarrafzadeh: Channel routing in knock-knee mode: simplified algorithms and proofs. Algorithmica 1, 213-221, 1986.

9. S. Muthukrishnan, M. Paterson, S.C. Sahinalp and T. Suel: Compact Grid Layouts of Multi-Level Networks. 31st ACM Symp. on Theory of Computing, 1999.

10. R.Y. Pinter, On routing two-point nets across a channel, 19th ACM-IEEE Design Automation Conf. (1982) 894-902.

11. A.L Rosenberg: Three-Dimensional VLSI: A Case Study. Journal of the ACM, 30(3), 1983, pp 397-416.

12. D.R. Wood: A New Algorithm and Open Problems in Three-Dimensional Orthogonal Graph Drawing. Proc. 10th Australasian Work. Combinatorial Algorithms, (AWOCA), pp. 157-167, 1999.

13. T.Yamada and S. Ueno: On Three-Dimensional Layout of De Bruijn Networks. 2002 IEEE International Symposium on Circuits and Systems (ISCAS '02), Vol. III, pp.779-782, 2002.

14. T.Yamada and S. Ueno: On Three-Dimensional Layout of Pyramid Networks. 2002 IEEE Asia Pacific Conference on Circuits and Systems (APCCAS '02), 2002.

15. C.-H. Yeh, B. Parhami, E.A. Varvarigos and H. Lee: VLSI Layout and Packaging of Butterfly Networks. ACM Symp. on Parallel Algorithms and Architectures (SPPA), pp. 196-205, 2000.

16. C.-H. Yeh, E.A. Varvarigos and B. Parhami: Multilayer VLSI Layout for Interconnection Networks. 2000 Int.l Conference on Parallel Processing (ICPP), pp. 33-40, 2002 . 